For Debate...

\title{
Immunisation of children by a nurse without a doctor present
}

\author{
NORMA JEFFERSON, GILLIAN SLEIGHT, AIDAN MACFARLANE
}

\section{Abstract}

Over 16 months 148 children were referred by health visitors and general practitioners to a specially trained nurse for failing to complete courses of immunisation. A further 91 children of travellers' families were identified as needing immunisation. The nurse carried out 810 immunisations on 237 of these children in their homes without a doctor being present. There were only two refusals, and one child suffered a mild anaphylactic shock. The cost per immunisation, in nurse's salary and travel expenses, was \&8.

This is an effective and fairly inexpensive way of achieving uptake of immunisation in such groups of children, and there seems no reason why trained nurses should not give immunisations either in a child health clinic or at home, without a doctor present.

\section{Introduction}

Immunisation in children is an effective primary preventive procedure, yet immunisation rates in Britain have for a long time fallen far short of ideal. ${ }^{1}$ Various methods of increasing the uptake of immunisation have been tried, including special information campaigns, computer controlled immunisation systems, the running of child health clinics at times suitable for parents, the increased availability of expert opinions on immunisation, and the re-education of those responsible for education about immunisation. Although some of these methods have increased the uptake of immunisation in certain groups, they have failed to reach two groups of parents and children: travelling families and those who consistently fail to attend clinics. These two groups might, however, be contacted successfully if a domiciliary immunisation service were established. Such a scheme has been shown to be effective $^{2}$ but also proved to be expensive, as it required $80 \%$ of a state enrolled nurse's working hours and 46 sessions of clinical medical officer time to immunise 156 children.

In Oxford city, because immunisation rates have traditionally been high (table), the children of travelling families and those who consistently fail to attend clinics were easily identifiable. We describe a local attempt to solve their immunisation problems with a domiciliary service run by an immunisation nurse.

District Department of Community Medicine, Oxfordshire Health Authority, Headington, Oxford OX3 9DZ

NORMA JEFFERSON, RGN, immunisation nurse

GILLIAN SLEIGHT, MB, BS, senior clinical medical officer

AIDAN MACFARLANE, BCHIR, FRCP, consultant community paediatrician

Correspondence to: Dr J A Macfarlane, Community Health Offices, Radcliffe Infirmary, Woodstock Road, Oxford.
Immunisation rates (\%) among children born in Oxford 1981-3

\begin{tabular}{lccc}
\hline & $\begin{array}{c}\text { Children born in 1981 } \\
\text { (course completed by } \\
\text { December 1983) }\end{array}$ & $\begin{array}{c}\text { Children born in 1982 Children born in 1983 } \\
\text { (course completed by } \\
\text { December 1984) }\end{array}$ & $\begin{array}{c}\text { (course completed by } \\
\text { December 1985) }\end{array}$ \\
Immunisation & 96 & 96 & 98 \\
\hline $\begin{array}{l}\text { Diphtheria/tetanus/polio } \\
\text { Pertussis }\end{array}$ & 76 & 84 & 86 \\
Measles & 86 & 87 & 94 \\
\hline
\end{tabular}

\section{Methods}

An immunisation nurse was contracted on a staff nurse's salary for 16 hours a week. Her salary, expenses, and milage reimbursement were paid for out of the medical budget for child health and amounted to $£ 4800$ a year.

Before starting work she undertook a one month training course, which consisted of weekly attendance at a child health clinic run by the senior medical officer responsible for immunisation in Oxfordshire(GS), attendance at a day course in resuscitation organised by the local ambulance service, supervised practical training in the giving of immunisation, a programme of reading about immunisation in children, and training by the higher clerical officer responsible for the administrative side of the recording of immunisations and the immunisation computer program. The subjects covered included the indications for and contraindications to immunisation, the care of vaccines, the keeping of records, health education, and how to deal with anaphylactic reactions. At the end of the course a certificate was given to verify that this training had been undertaken. The doctors supervising the course, the administrative nursing staff, and the nurse herself thus accepted that she was now competent to give immunisations without a doctor being present.

It was decided, in view of the number of working hours available, that the nurse should cover Oxford city, which has a total population of around 100000 and a preschool population of 6500 . Health visitors and school nurses were instructed to refer children who regularly did not attend child health clinics for immunisation but for whom a consent form had been signed by the parents and the prescription written by a doctor. In addition, those in need of immunisation were identified by direct visits to the travelling families in either the two permanent travellers' camps in Oxford city or various illegal roadside sites, as and when they were identified.

\section{Results}

The results presented here cover the first 16 months of the project from January 1985 to April 1986. The subjects fell into two distinct groups: those who consistently failed to attend clinics and travelling families. In all, 148 children were referred by health visitors and doctors. The main reasons for failure to start or complete courses of immunisation (as stated to the immunisation nurse) were: (a) the family was misinformed, $(b)$ the family was socially deprived and unable to attend clinic, and $(c)$ the child suffered from frequent minor ailments. Underlying many of these reasons was the fear of immunisation, which was considerably allayed by the opportunity to have the child immunised at home.

In these 148 children the following numbers of immunisations were given: 148 diphtheria and tetanus, 145 polio, 106 pertussis, 96 measles, and two diphtheria alone. Two sets of parents refused all immunisations for their children. The age range of children who underwent immunisation in this group was 3 months to 11 years.

In all, 91 travellers' children were seen. The following numbers of 
immunisations were given: 121 diphtheria and tetanus, nine pertussis, 130 polio, 45 measles, and eight rubella. The age range covered was 3 months to 18 years. In addition, five adult women received oral polio vaccine.

A total of 810 immunisations were carried out on 237 children at a cost of around $£ 8$ per immunisation in nursing time and travel payments. During the study the nurse completed immunisation up to the thirteenth month of . the course in 73 children referred by health visitors and in 27 travellers' children.

\section{Discussion}

Nurses have been discouraged from performing immunisations in the absence of a doctor on the grounds that a doctor should be present in case an anaphylactic reaction occurs. A recent study indicated, however, that such problems actually arise extremely rarely, ${ }^{2}$ and from this it may be estimated that a nurse giving 100 immunisations a week will encounter a severe anaphylactic reaction once in 18 years. Furthermore, there is no evidence that a well trained nurse is less competent in dealing with the anaphylactic reaction than a well trained doctor. Therefore, with the agreement of the nurse concerned and those administratively responsible for the services, there seem to be no contraindications to a nurse giving immunisations without a doctor being present.

It had originally been feared that there would be an upsurge of unnecessary requests for the service as soon as it was known about, but this did not happen. Any general health problems that were discovered as a result of the contact between the immunisation nurse and a family were referred back to the family's own health visitor, except in the case of the travellers' families, who were not registered with a health visitor. All health visitors seemed happy to refer families to the immunisation nurse, as in most cases the health visitor had already visited many times to persuade the family to have immunisations.

Of the 237 children who underwent immunisation, only one suffered a mild vasovagal attack. In eight cases further medical advice about the advisability of immunisation was sought, and in all these cases the children were considered to be suitable for immunisation. Two children with congenital heart disease had been advised not to attend child health clinics for immunisation because of the risk of infection. There were four children with other handicaps who had previously been advised not to undergo immunisation, all of whom were, on review, considered to be suitable for immunisation.

In the case of those who persistently failed to attend clinics and the travelling families much preliminary work had to be done, not only to gain the confidence of the families but also to convince the health visitors and the clinic doctors of the value of the service. This was done by visiting surgeries and clinics and by spending time identifying clinic defaulters. Travelling families in particular tend to view outside intervention suspiciously, and this was reflected in their initially slow uptake of the service in the first six months of the project, but once the nurse became trusted and widely known the families were keen to make use not only of the immunisations but also of the nurse's general medical advice. By the end of the study the nurse was carrying out about 15 immunisations a week. The number of children among the travelling families who were immunised against pertussis was extremely low, as travelling families do not usually allow their children to have the whooping cough vaccine because of entrenched fear and mythology. One extended family, however, who had until then used a blue ribbon worn round the neck to protect the children against whooping cough, did allow them to be immunised.

In her work with the travellers the immunisation nurse not only gave immunisations but offered a service similar to that of a general health worker. Thus she also registered families with general practitioners; arranged schooling for the children by gaining places in local schools or arranging visits from the mobile bus; liaised with the social services over children at risk; organised family planning advice and antenatal screening for mothers with histories of stillbirth and spina bifida; and arranged for sick children to be admitted to hospital. In a typical week the nurse spent about seven hours with travellers, four hours with those who persistently failed to attend clinics, two hours informing health visitors and other workers about immunisations, and two doing administrative work.

The immunisation nurse was also often referred to see one child in a family but in fact immunised several children in the same family who had previously failed to start or complete a course of immunisation.

The cost of each immunisation in the study in nursing time and travel was about $£ 8$, compared with about $£ 14$ for the central Manchester domiciliary scheme. ${ }^{3}$ If, however, the calculation is based on the immunisation rate of 15 a week, achieved towards the end of the study, the cost falls to about £6. This may also be compared with the fee for item of service of about $£ 3$ for each immunisation that is paid to general practitioners or the cost of immunisation in a child health clinic attended by a clinical medical officer and a health visitor performing about 20 immunisations in a session, which works out at about $£ 2$ per immunisation, though none of these costings takes into account the giving of advice, the developmental examinations, etc, that often take place at the same time as immunisation.

We therefore propose that to obtain maximum immunisation coverage of a population of preschool children cost effectively and efficiently most immunisations should be carried out at child health clinics and the few vulnerable children who fail to attend child health clinics may be immunised at home, still at relatively low cost, by an immunisation nurse. There seems to be no reason why suitably trained nurses should not give immunisations without a doctor being present.

\section{References}

1 Anonymous. Failure to vaccinate [Editorial]. Lancet 1983;ii:1343.

2 James AE. Domiciliary immunisation for preschool child defaulters. Br Med $\mathcal{F}$ 1984;289:1429-31. 3 Pollock TN, Morris J. A 7 year survey of disorders attributed to vaccinations in North West Thames region. Lancet 1983;i:753-7.

(Accepted 3 December 1986)

Physiotherapists and occupational therapists often work with handicapped children, some of whom are known carriers of the cytomegalovirus. What are the risks to the therapist and should they have any specific regular blood tests?

Infection of adults with cytomegalovirus only rarely causes a clinically apparent illness, usually a glandular fever that is self limiting. If a pregnant woman has a primary infection, however, intrauterine infection will occur in $40-50 \%$ of pregnancies. Of fetuses infected, approximately $5 \%$ will have severe congenital infection and a further $10-15 \%$ may have some complication later in life. Reactivation of the virus in a woman shown to have been infected before pregnancy may also result in intrauterine infection but the fetus is unlikely to be affected. Cytomegalovirus infection is common in the community with approximately $\mathbf{5 0 - 9 0 \% ~ ( d e p e n d e n t ~ o n ~ e t h n i c ~ a n d ~ s o c i o - ~}$ economic group) of pregnant women having been infected earlier in life. Approximately $1 \%$ of women will have a primary cytomegalovirus infection during pregnancy. Whether this rate of infection is increased in health care workers having close contact with neonates, infants, and children is a matter of debate. ${ }^{12}$ Several studies in the United States on the increased risk to health care workers have produced conflicting results so there is no consensus of advice for preventing cytomegaloviral infection. ${ }^{2}$ If there is an increased risk it would not appear to be sufficient to warrant the cost and organisation required for repeated serological testing, even if the health care worker is pregnant. Routine screening on employment is not recommended as it is not known whether advising less contact at work with infants and children would reduce the risk of primary cytomegalovirus in the susceptible pregnant health care worker. This advice would be reviewed if a safe and effective vaccine were to become available. Attention to hand washing after patient contact is advisable, however, and it would be prudent for pregnant physiotherapists and occupational therapists to avoid close contact with children known to be excreting cytomegalovirus.-P MORGAN-CAPNER, consultant virologist, Preston. 1 Kinney JS, Onorato IM, Stewart JA, et al. Cytomegaloviral infection and disease. F Infect Dis 1985;
151:772-4.

2 Plotkin SA. Cytomegalovirus in hospitals. Pediatr Infect Dis 1986;5:177-8. 